\title{
gr/gr deletion predisposes to testicular germ cell tumour independently from altered spermatogenesis: results from the largest European study
}

\author{
Daniel Moreno-Mendoza ${ }^{1} \cdot$ Elena Casamonti $^{2} \cdot$ Donatella Paoli $^{3} \cdot$ Chiara Chianese $^{1} \cdot$ Antoni Riera-Escamilla $^{1}$. \\ Claudia Giachini ${ }^{2} \cdot$ Maria Grazia Fino ${ }^{2}$ - Francesca Cioppi ${ }^{2} \cdot$ Francesco Lotti $^{2} \cdot$ Serena Vinci $^{2} \cdot$ Angela Magini $^{2}$. \\ Elisabet Ars $^{4} \cdot$ Josvany Sanchez-Curbelo $^{1} \cdot$ Eduard Ruiz-Castane $^{1} \cdot$ Andrea Lenzi $^{3} \cdot$ Francesco Lombardo $^{3}$. \\ Csilla Krausz ${ }^{1,2}$
}

Received: 27 December 2018 / Revised: 26 March 2019 / Accepted: 13 April 2019 / Published online: 3 May 2019

(c) European Society of Human Genetics 2019

\begin{abstract}
The association between impaired spermatogenesis and TGCT has stimulated research on shared genetic factors. Y chromosome-linked partial AZFc deletions predispose to oligozoospermia and were also studied in TGCT patients with controversial results. In the largest study reporting the association between $g r / g r$ deletion and TGCT, sperm parameters were unknown. Hence, it remains to be established whether this genetic defect truly represents a common genetic link between TGCT and impaired sperm production. Our aim was to explore the role of the following Y chromosome-linked factors in the predisposition to TGCT: (i) $g r / g r$ deletion in subjects with known sperm parameters; (ii) other partial AZFc deletions and, for the first time, the role of partial AZFc duplications; (iii) DAZ gene dosage variation. 497 TGCT patients and 2030 controls from two Mediterranean populations with full semen/andrological characterization were analyzed through a series of molecular genetic techniques. Our most interesting finding concerns the $\mathrm{gr} / \mathrm{gr}$ deletion and $D A Z$ gene dosage variation (i.e., $D A Z$ copy number is different from the reference sequence), both conferring TGCT susceptibility. In particular, the highest risk was observed when normozoospermic TGCT and normozoospermic controls were compared $(\mathrm{OR}=3.7 ; 95 \%$ $\mathrm{CI}=1.5-9.1 ; p=0.006$ for $\mathrm{gr} / \mathrm{gr}$ deletion and $\mathrm{OR}=1.8 ; 95 \% \mathrm{CI}=1.1-3.0 ; p=0.013$ for $D A Z$ gene dosage alteration). We report in the largest European study population the predisposing effect of $g r / g r$ deletion to TGCT as an independent risk factor from impaired spermatogenesis. Our finding implies regular tumour screening/follow-up in male family members of TGCT patients with $g r / g r$ deletion and in infertile $g r / g r$ deletion carriers.
\end{abstract}

These authors contributed equally: D. Moreno Mendoza, E. Casamonti

Supplementary information The online version of this article (https:// doi.org/10.1038/s41431-019-0420-7) contains supplementary material, which is available to authorized users.

Csilla Krausz

c.krausz@dfc.unifi.it

1 Andrology Department, Fundació Puigvert, Universitat Autònoma de Barcelona, Instituto de Investigaciones Biomédicas Sant Pau (IIB-Sant Pau), Barcelona, Spain

2 Department of Experimental and Clinical Biomedical Sciences "Mario Serio", Centre of Excellence DeNothe, University of Florence, Florence, Italy

\section{Introduction}

Testicular germ cell tumour (TGCT) is the most common malignant neoplasia in reproductive age [1]. The worldwide incidence of TGCTs shows significant variation between different geographical regions and ethnic groups. In Europe it ranges from 1.6 to 9.9 cases per 100,000 individuals [1]. These tumours are derived from germ cell neoplasia in situ

3 Laboratory of Seminology-Sperm Bank "Loredana Gandini”, Department of Experimental Medicine "Sapienza", University of Rome, Rome, Italy

4 Molecular Biology Laboratory, Fundació Puigvert, Instituto de Investigaciones Biomédicas Sant Pau (IIB Sant Pau), Universitat Autònoma de Barcelona, Barcelona, Spain 
(GCNIS) cells, which are arrested and transformed fetal gonocytes that failed to mature to spermatogonia [2]. Data in the literature indicate that both environmental and genetic factors acting on the primordial germ cells/gonocytes are implicated in the etiopathogenesis of this tumour [2-5].

For instance, familiarity and ethnic differences in TGCT incidence indicate a strong genetic component. Studies in families demonstrated that the familial risk of TGCT is 8-fold to 10-fold elevated for brothers of affected men and 4-fold to 6-fold elevated for fathers/sons of cases, compared with the general male population [6, 7]. In addition, it is well known that Asian and African populations have a low risk of TGCTs and men with African or Asian descent maintains their low risk even living in a geographic area of high risk [4, 8]. Based on population and genomic data Litchfield et al. [9] estimated the heritability of TGCT to be in a consistent range of $37-49 \%$.

Besides genetic factors, a number of clinical predisposing factors are known. Among them, the presence of the components of the "testicular dysgenesis syndrome" [10], such as cryptorchidism, testicular hypotrophy/infertility, hypospadias and microlithiasis, confer higher risk for TGCT [11, 12].

Given the association between infertility and TGCT, genetic anomalies related to infertility has been studied as potential predisposing factors to TGCT.

The human Y chromosome harbors genes essential for testis development and function, such as $S R Y$, which is indispensable for testis determination, and the genes residing in the azoospermia factor regions (AZFa, $\mathrm{AZFb}$, and $\mathrm{AZFc}$ ), which are involved in spermatogenesis [13]. AZF microdeletions are a well-established genetic cause of severe spermatogenic failure $(5-10 \%$ of azoospermic and $2-5 \%$ of severe oligozoospermic men) [14]. Among the partial AZFc deletions, the clinically most relevant one is called $g r / g r$, discovered by Repping et al. [15]. This deletion removes half of the gene content and represents a significant risk factor for spermatogenic impairment. However, the entity of the risk for a $g r / g r$ deletion carrier to be affected by oligozoospermia varies between different ethnicities and shows the highest values in the Mediterranean area: 5.8 (CI 2.0-16.9, $p=0.001$ ) and $2.9(1.0-8.0)$ in the Italian and Spanish ethnicity, respectively [13].

Three studies focused on the role of complete AZF deletions in TGCT, but a relationship with this disease could not be proven [16-18]. No association was found between increased risk of TGCT and Y haplogroups neither [19-21]. Concerning the partial AZFc deletions, the role of $\mathrm{gr} / \mathrm{gr}$ deletions in TGCTs is still debated. In fact, only one out of three published studies observed an increased risk. Indeed, a large multicenter study by Nathanson and collaborators [22] show a 2-fold and 3-fold increased risk for sporadic and familiar TGCT in subjects carrying the $g r / g r$ deletion, respectively. The two subsequent studies based on relatively small cohorts from England and Italy did not confirm the relationship between $g r / g r$ deletions and TGCT [21, 23]. Worth noting that only one of the three studies considered sperm parameters in TGCT patients [21]. Due to the lack of information on the semen phenotype in cancer patients carrying the $g r / g r$ deletion, it remains to be established whether this genetic defect truly represents a common genetic link between TGCT and impaired sperm production.

Only a single manuscript addressed the role of other partial AZFc deletions and in this study no correlation was found with TGCT [23].

We aimed at providing a comprehensive characterization of partial AZFc rearrangements in the largest European case-control study population available to date. In addition, we have addressed an important clinical question concerning the role of $g r / g r$ deletion in TGCT in relationship with the semen phenotype.

\section{Materials}

\section{Subjects}

We prospectively evaluated 497 patients with TGCT and 2030 control subjects without TGCT who were referred to the University Hospital of Careggi (Italy), Fundació Puigvert (Spain) and University of Rome "La Sapienza" (Italy). The control group included 1068 normozoospermic and 962 non-normozoospermic men with no history of TGCT, no family history of TGCT, no history of cryptorchidism or other abnormal andrological findings and no genetic abnormalities (karyotype alterations and complete AZF deletions). The subjects belonging to these two control groups have been studied previously in relationship with male infertility (for review see Krausz and Casamonti [13]); for this study 24 novel normozoospermic and 18 nonnormozoospermic controls were added. TGCT patients came to visit for semen cryopreservation before chemo/ radiotherapy. Semen samples were collected by masturbation after 3-5 days of sexual abstinence and semen parameters were investigated according to the WHO guidelines (World Health Organization) [24]. A complete clinical history and andrological exams (including physical examination, scrotal ultrasound and hormonal analysis) were performed. Among TGCT patients, 353 (71\%) were affected by seminoma, while 144 (29\%) were affected by nonseminoma. Fifty-seven TGCT patients had history of cryptorchidism, 3 had hypospadias and 4 had family history of TGCT. Overall, 268 (54\%) TGCT subjects were normozoospermic (total sperm count $\left.\geq 39 \times 10^{6}\right)$ and $229(46 \%)$ were non-normozoospermic (total sperm count $<39 \times 10^{6}$ ). Among the latter, 35 were affected by azoospermia. This study was approved by local ethics committees and all participants provided written informed consent. 


\section{Methods}

\section{Molecular analysis}

We used standard salting-out method to extract genomic DNA from peripheral blood samples [25]. The analysis for Y chromosome AZF microdeletions was performed by multiplex PCR (using markers from AZFa, AZFb, and $A Z F c$ regions according to the EAA/EMQN [14]). The screening for partial AZFc rearrangements was performed in Florence and Barcelona centers by using a multistep method described by Giachini et al. [26]. The first step consisted in multiplex PCR plus/minus based on the amplification of Specific sequence-tagged sites (STSs): sY1291 and sY1191. We identified the $g r / g r$ deletion by the absence of sY1291. The $b 2 / b 3$ deletion is characterized by the absence of sY1191 and the $b 1 / b 3$ deletion by the absence of sY1291 and of sY1191. To confirm the multiplex PCR results, we repeated the analysis in all samples with suspected deletion by using a simplex PCR for sY1291 and sY1191. In addition, two subsequent confirmatory steps have been performed in each deletion carrier (as described in Giachini et al. [26].): (i) gene dosage analysis; (ii) RFLP analysis. All the partial AZFc rearrangements identified in the present study were submitted in the LOVD v.3.0 database, https://databases.lovd.nl/shared/variants (variant IDs 461227-461232).

\section{AZFc gene dosage}

$C D Y 1$ and $D A Z$ gene dosage was performed in all TGCT patients and in 1424 control subjects in order to confirm gene-copy loss in deletion carriers and to define gene duplications in the AZFc region. In brief, we simultaneously amplified the AZFc locus to be quantified (CDY1 and DAZ) and a homologous locus outside the AZFc interval (CDY2 and $D A Z L$, respectively) as an internal standard with a known number of copies, using a single primer pair in a Quantitative Fluorescence-PCR (QF-PCR) reaction with the maximum of 24 cycles (end point of exponential phase). The fluorescence PCR products were separated on automatic sequencer (ABI PRISM ${ }^{\circledR} 3730$ XL DNA Sequencer, Applied Biosystem, Milan, Italy). For copy number quantification we compared the peak area corresponding to the $C D Y 1$ and $D A Z$ loci to their homologs $C D Y 2$ and $D A Z L$, respectively.

\section{RFLP analysis}

For $D A Z$ qualitative analysis, we chose the sequence family variant (SFV) at STS sY587 in intron 10, which discriminates $D A Z 1 / 2$ from $D A Z 3 / 4$. For $C D Y 1$, we used a SFV situated $7750 \mathrm{bp}$ upstream of the $C D Y 1$ translation start codon (CDY7750), which distinguishes CDY1A from $C D Y 1 B$. SFV s were scored by PCR followed by enzyme digestion using the following enzymes: DraI for $D A Z$ sY587 and PvuII for CDY1-7750.

\section{Y chromosome haplogroups definition}

Subjects with partial AZFc deletions were genotyped for six binary markers (M168, M145, M9, M45, M96, LLy22g). In brief, multiplex PCR of the regions spanning the six binary makers was followed by enzymatic clean-up of PCR products and multiplex single-base primer extension (SNaPshot Multiplex System, Applied Biosystems) [27]. DNA purification and analysis of primer extension products by ABI PRISM ${ }^{\circledR} 3730$ XL DNA Sequencer. The combination of genotypes allowed us defining specific clades or haplogroup clusters: (i) $\mathrm{AB}$; (ii) $\mathrm{DE}^{*} \mathrm{DE}$; (iii) CFGHI or J; (iv) E; (v) KLMNO*OST; (vi) N1; (vii) $\mathrm{P}^{*} \mathrm{QR}$ (see Karafet et al. [28]). A single PCR of the 12f2 marker was performed to differentiate the $\mathrm{J}$ haplogroup from the CFGHI cluster.

\section{Statistical analysis}

SPSS software (version 25.0 Chicago, IL, USA) was used. We tested the significance of the observed differences in the incidence of the AZFc rearrangements between the TGCT patients and the control group using Fisher's exact test: our null hypothesis was that the incidence is the same in the TGCT patients and in the control group. For comparisons of medians between groups of different genotypes, the nonparametric Mann-Whitney $U$-test for independent samples was applied. After Bonferroni correction considering two independent variables, (i.e., deletions and duplications), a $p$-value $\leq 0.025$ was considered statistically significant for each test.

\section{Results}

\section{Analysis of complete AZF deletions and partial AZFc rearrangements in TGCT patients}

In this study we aimed at: (i) defining the frequency of complete AZF deletions, partial AZFc deletions, partial AZFc duplications and $D A Z$ dosage alteration; (ii) providing a detailed molecular and phenotypical characterization of the rearrangements carriers.

\section{Complete AZF deletions}

None were found in the entire cohort of TGCT patients $(n=497)$. 


\section{Partial AZFc deletions}

14/497 patients (2.8\%) carried the $g r / g r$ deletion (chrY:g. (24876071_25505070)_(25505734_25316178)del) (hg19) (LOVD3 DB-ID:chrY_000067, ID Variant:0000461229), whereas b2/b3 (chrY: g.(24524070_24872541)_ (24876071_25505070)del) (hg19) (LOVD3 DB-ID: chrY_000068, ID Variant: 0000461230), b1/b3 (chrY: g.(23978074_24354700)_(25316178_25505734)del (hg19) (LOVD3 DB-ID: chrY_000135, ID Variant: 0000461228) and atypical (chrY: g.(25505596_26191376)_(26194116_ 26979967) del) (hg19) (LOVD3 DB-ID: chrY_000134, ID Variant: 0000461227) deletions were found in only 5 subjects $(1.0 \%)$ with a frequency of $0.6 \%, 0.2 \%$, and $0.2 \%$, respectively (Table 1 ).

1) $\mathrm{gr} / \mathrm{gr}$ deletion: this deletion type was more frequent in normozoospermic compared to non-normozoospermic TGCT patients (3.4\% vs. $2.2 \%)$, and in seminoma $v s$. non-seminoma patients $(3.1 \%$ vs. $2.1 \%)$ without reaching statistical significance $(p=0.305$ and $p=0.385$, respectively). The highest frequency was observed in normozoospermic seminoma patients $(4.1 \%)$ (Table 1$)$.

The histology and semen phenotypes together with the $g r / g r$ deletion subtypes for the $14 g r / g r$ deletion carriers are reported in Table 2. As for histology, 78.6\% were seminomas, whereas only $21.4 \%$ were non-seminomas. Only 2 patients (14.3\%) were azoospermic, 3 (21.4\%) were oligozoospermic and the large majority were normozoospermic $(64.3 \%)$. The combined gene dosage and RFLP analysis allowed a more detailed molecular characterization of the deletion subtypes based on the number and type of $C D Y 1$ and $D A Z$ copies. All patients, except one, showed a reduced $C D Y 1$ and $D A Z$ gene copy number in respect to the reference sequence, i.e., 1 copy of $C D Y 1$ and 2 copies of $D A Z$. The increased $C D Y 1$ and $D A Z$ copy number in one patient was due to deletion followed by multiple duplications. In fact, RFLP analysis confirmed the presence of $3 C D Y 1 A$ and $6 D A Z 3 / 4$ copies. This subject was affected by non-seminoma and the semen phenotype was severe oligozoospermia.

Based on the type of the deleted $C D Y 1$ and $D A Z$ copies, four different subtypes were identified: $C D Y 1 A+D A Z 3 / 4$ (7/14, 50\%), CDY1A+DAZ1/2 (4/14, 28.6\%), CDY1B $+D A Z 1 / 2(2 / 14,14.3 \%)$, and CDY1B+DAZ3/4 (1/14, $7.1 \%)$. Summing frequencies up, $C D Y 1 A$ was missing in 11 (78.6\%), while $C D Y 1 B$ was missing only in $3(21.4 \%)$ patients. Concerning $D A Z$ copy number, $D A Z 3 / 4$ was deleted in $8(57.1 \%)$ and $D A Z 1 / 2$ in $6(42.9 \%)$ subjects. All oligo/azoospermic patients affected by seminoma were lacking CDY1A whereas those with normozoospermia had CDY1A copy deletion in $66.7 \%$. Altogether, no relationship was found between the type of deleted gene copy and the cancer histology or the semen parameters.

The analysis of the Y chromosome haplogroups in the deletion carriers did not reveal a specific haplogroup associated with $g r / g r$ deletion. The most frequent haplogroup in our cohort was $\mathrm{P}^{*} \mathrm{QR}(36 \%)$. No differences were observed in haplogroup distribution in relationship with the semen phenotype (Table 2).

2) $b 2 / b 3, b 1 / b 3$ and atypical deletions: similar to $g r / g r$, these deletions were slightly more frequent in
Table 1 Frequency of AZFc rearrangements and $D A Z$ dosage alterations in TGCT patients (reported in subgroups based on histotypes and total sperm count) and in controls (reported in subgroups based on total sperm count)

\begin{tabular}{|c|c|c|c|c|c|}
\hline & & $g r / g r$ deletion & $\begin{array}{l}\text { Other partial } \\
\text { AZFc deletions }\end{array}$ & $\begin{array}{l}\text { Partial AZFc } \\
\text { duplications }\end{array}$ & $\begin{array}{l}D A Z \text { dosage } \\
\text { alteration }\end{array}$ \\
\hline \multirow[t]{9}{*}{ TGCT } & Total & $14 / 497(2.8 \%)$ & $5 / 497(1.0 \%)$ & $22 / 478(4.6 \%)$ & $51 / 497(10.3 \%)$ \\
\hline & $\mathrm{NZ}$ & $9 / 268(3.4 \%)$ & $3 / 268(1.1 \%)$ & $9 / 256(3.5 \%)$ & $28 / 268(10.4 \%)$ \\
\hline & NNZ & $5 / 229(2.2 \%)$ & $2 / 229(0.9 \%)$ & $13 / 222(5.9 \%)$ & $23 / 229(10.0 \%)$ \\
\hline & Seminoma & $11 / 353(3.1 \%)$ & $4 / 353(1.1 \%)$ & $16 / 338(4.7 \%)$ & $38 / 353(10.8 \%)$ \\
\hline & Non-seminoma & $3 / 144(2.1 \%)$ & $1 / 144(0.7 \%)$ & $6 / 140(4.3 \%)$ & $13 / 144(9.0 \%)$ \\
\hline & Seminoma NZ & $8 / 196(4.1 \%)$ & $2 / 196(1.0 \%)$ & $7 / 186(3.8 \%)$ & $23 / 196(11.7 \%)$ \\
\hline & Seminoma NNZ & $3 / 157(1.9 \%)$ & $2 / 157(1.3 \%)$ & $9 / 152(5.9 \%)$ & $15 / 157(9.5 \%)$ \\
\hline & $\begin{array}{l}\text { Non- } \\
\text { Seminoma NZ }\end{array}$ & $1 / 72(1.4 \%)$ & $1 / 72(1.4 \%)$ & $2 / 70(2.9 \%)$ & $5 / 72(6.9 \%)$ \\
\hline & $\begin{array}{l}\text { Non- } \\
\text { Seminoma NNZ }\end{array}$ & $2 / 72(2.8 \%)$ & $0 / 72(0.0 \%)$ & $4 / 70(5.7 \%)$ & $8 / 72(11.1 \%)$ \\
\hline \multirow[t]{3}{*}{ Controls } & Total & $40 / 2030(2.0 \%)$ & $11 / 2030(0.5 \%)$ & $51 / 1373(3.7 \%)$ & $111 / 1424(7.8 \%)$ \\
\hline & NZ & $10 / 1068(0.9 \%)$ & $2 / 1068(0.2 \%)$ & $27 / 756(3.6 \%)$ & $46 / 768(6.0 \%)$ \\
\hline & NNZ & $30 / 962(3.1 \%)$ & $9 / 962(0.9 \%)$ & $24 / 617(3.9 \%)$ & $65 / 656(9.9 \%)$ \\
\hline
\end{tabular}

The columns report the number of subject observed with rearrangements/total subject patients and the percentage in parenthesis

$N Z$ normozoospermic, $N N Z$ non-normozoospermic

${ }^{a}$ Other partial AZFc deletions refers to $b 2 / b 3, b 1 / b 3$, and atypical deletion 
Table 2 Phenotype/genotype description of the 14 TGCT patients with $g r / g r$ deletion and of the 5 TGCT patients with other partial AZFc deletions

\begin{tabular}{|c|c|c|c|c|c|c|c|c|c|c|}
\hline \multirow[t]{2}{*}{ Patient } & \multirow[t]{2}{*}{ Origin } & \multirow{2}{*}{$\begin{array}{l}\text { Testis tumor } \\
\text { histology }\end{array}$} & \multirow{2}{*}{$\begin{array}{l}\text { Total } \\
\text { sperm } \\
\text { count } \\
\left(10^{6}\right)\end{array}$} & \multirow{2}{*}{$\begin{array}{l}\text { Y chromosome } \\
\text { clade/ } \\
\text { haplogroups } \\
\text { cluster }\end{array}$} & \multicolumn{2}{|c|}{ PCR plus/minus } & \multicolumn{2}{|c|}{$C D Y 1 / D A Z$ dosage } & \multicolumn{2}{|l|}{ RFLP } \\
\hline & & & & & sY1191 & sY1291 & $\begin{array}{l}C D Y 1 \\
\text { copy number }\end{array}$ & $\begin{array}{l}D A Z \\
\text { copy number }\end{array}$ & $\begin{array}{l}C D Y 1 \\
\text { deleted copy }\end{array}$ & $\begin{array}{l}D A Z \\
\text { deleted copy }\end{array}$ \\
\hline \multicolumn{11}{|l|}{$g r / g r$ deletion } \\
\hline A818 & Italian & Non-seminoma & 0.00 & $\mathrm{~J}$ & Present & Absent & 1 & 2 & $C D Y 1 A$ & $D A Z 3 / 4$ \\
\hline A1171 & Italian & Seminoma & 720.00 & $\mathrm{P}^{*} \mathrm{QR}$ & Present & Absent & 1 & 2 & $C D Y 1 A$ & $D A Z 1 / 2$ \\
\hline A1445 & Italian & Seminoma & 244.80 & $\mathrm{P}^{*} \mathrm{QR}$ & Present & Absent & 1 & 2 & $C D Y 1 A$ & $D A Z 3 / 4$ \\
\hline A1663 & Italian & Non-seminoma & 7.56 & CFGHI & Present & Absent & 3 & 6 & $C D Y 1 B$ & $D A Z 1 / 2$ \\
\hline A1713 & Italian & Seminoma & 109.20 & $\mathrm{P}^{*} \mathrm{QR}$ & Present & Absent & 1 & 2 & $C D Y 1 A$ & $D A Z 3 / 4$ \\
\hline A1734 & Italian & Seminoma & 0.36 & CFGHI & Present & Absent & 1 & 2 & $C D Y 1 A$ & $D A Z 3 / 4$ \\
\hline A1912 & Italian & Seminoma & 209.00 & $\mathrm{E}$ & Present & Absent & 1 & 2 & $C D Y 1 B$ & $D A Z 3 / 4$ \\
\hline A2140 & Italian & Seminoma & 240.00 & $\mathrm{P}^{*} \mathrm{QR}$ & Present & Absent & 1 & 2 & $C D Y 1 B$ & $D A Z 1 / 2$ \\
\hline KG8 & Italian & Non-seminoma & 152.00 & KLO & Present & Absent & 1 & 2 & $C D Y 1 A$ & $D A Z 3 / 4$ \\
\hline KG62 & Italian & Seminoma & 56.00 & KLO & Present & Absent & 1 & 2 & $C D Y 1 A$ & $D A Z 1 / 2$ \\
\hline KG162 & Italian & Seminoma & 35.00 & $\mathrm{~J}$ & Present & Absent & 1 & 2 & $C D Y 1 A$ & $D A Z 1 / 2$ \\
\hline $16-001$ & Spanish & Seminoma & 98.70 & $\mathrm{E}$ & Present & Absent & 1 & 2 & $C D Y 1 A$ & $D A Z 3 / 4$ \\
\hline $17-484$ & Spanish & Seminoma & 89.60 & $\mathrm{~J}$ & Present & Absent & 1 & 2 & $C D Y 1 A$ & $D A Z 3 / 4$ \\
\hline $17-733$ & Spanish & Seminoma & 0.00 & $\mathrm{P} * \mathrm{QR}$ & Present & Absent & 1 & 2 & $C D Y 1 A$ & $D A Z 1 / 2$ \\
\hline \multicolumn{11}{|c|}{ Other partial AZFc deletions: } \\
\hline \multicolumn{11}{|c|}{$b 2 / b 3$ deletion } \\
\hline A1881 & Italian & Seminoma & 36.00 & CFGHI & Absent & Present & 2 & 4 & $C D Y 1 A$ & $D A Z 3 / 4$ \\
\hline KG117 & Italian & Seminoma & 38.40 & $\mathrm{E}$ & Absent & Present & 1 & 2 & $C D Y 1 B$ & $D A Z 3 / 4$ \\
\hline KG34 & Italian & Seminoma & 135.00 & CFGHI & Absent & Present & 1 & 2 & $C D Y 1 A$ & $D A Z 3 / 4$ \\
\hline \multicolumn{11}{|c|}{$b 1 / b 3$ deletion } \\
\hline KG156 & Italian & Seminoma & 40.00 & CFGHI & Absent & Absent & 2 & 4 & No deletion & $D A Z 1 / 2$ \\
\hline \multicolumn{11}{|c|}{ Atypical deletion } \\
\hline A1623 & Italian & Non-seminoma & 45.51 & $\mathrm{~J}$ & Present & Present & 1 & 4 & $C D Y 1 B$ & No deletion \\
\hline
\end{tabular}

normozoospermic compared to non-normozoospermic TGCT patients ( $1.1 \%$ vs $0.9 \%)$, and in seminoma (1.1\%) than in non-seminoma patients $(0.7 \%)$. The highest frequencies were observed in normozoospermic nonseminoma patients $(1.4 \%)$ and non-normozoospermic seminoma (1.3\%) (Table 1).

The molecular characterization and the associated semen and histology phenotypes of the 5 patients carrying the $b 2 /$ $b 3, b 1 / b 3$ and atypical deletions are reported in Table 2 . All patients with $b 2 / b 3$ deletion had seminoma. Two patients (66.7\%) were non-normozoospermic. Two patients showed reduced $C D Y 1$ and $D A Z$ gene copy number (1 CDY1 copy and $2 D A Z$ copies), the other patient presented $2 C D Y 1$ and $4 D A Z$ copies as a consequence of partial deletion followed by duplication, i.e., RFLP analysis revealed the deletion of the $C D Y 1 A$ and $D A Z 3 / 4$ copies. The three $b 2 / b 3$ deletion carriers showed a heterogeneous situation concerning the type of deleted $C D Y 1$ copy: $C D Y 1 A$ was deleted in 2 patients and $C D Y 1 B$ in 1 patient. Interestingly enough, all patients presented a deletion of $D A Z 3 / 4$ copy. The $b 1 / b 3$ deletion carrier had seminoma with normozoospermia and presented $2 C D Y 1$ copies and $4 D A Z$ copies (deletion of $D A Z 1 / 2$ followed by duplication). The patient with atypical deletion had non-seminoma and was normozoospermic and he showed $C D Y 1 B$ copy deletion without $D A Z$ copy deletion, i.e., presenting $D A Z 1 / 2$ and $D A Z 3 / 4$ copies.

Concerning the $\mathrm{Y}$ haplogroup analysis, it revealed that 3 out of 5 patients belong to the CFGHI cluster (60\%) whereas the remaining two presented haplogroup $\mathrm{E}$ and $\mathrm{J}$, respectively (Table 2 ).

\section{Partial AZFc duplications}

(Chry:g.(24876071_25505070)_(25505734_ 25316178)dup) (hg19) (LOVD3 DB-ID: chrY_000086, ID Variant: 0000461232); (chrY:g.(24524070_24872541)_(25316578_ 28457316)dup) (hg19) (LOVD3 DB-ID: chrY_000074, ID Variant: 0000461231): these rearrangements are defined when both $D A Z$ and $C D Y 1$ present increased copy number and it was observed in 22 TGCT patients $(4.6 \%)$. We 
identified patients with 3 or 4 copies of $C D Y 1$ and 6 or 8 copies of DAZ. Partial AZFc duplications were observed more frequently in non-normozoospermic compared to normozoospermic TGCT patients $(5.9 \%$ vs $3.5 \%)$ and in seminoma $(4.7 \%)$ vs. non-seminoma patients $(4.3 \%)$, without reaching statistical significance $(p=0.159$ and $p=0.523$, respectively). The highest frequency was observed in nonnormozoospermic seminoma patients (5.9\%) (Table 1).

\section{DAZ dosage alteration}

We defined it as any rearrangement (partial AZFc deletion or duplications or isolated $D A Z$ duplication) causing an alteration in $D A Z$ copy number from the canonical 4 copies (greater or less). In the 497 patients with TGCT, 51 (10.3\%) presented alterations in $D A Z$ dosage. $D A Z$ dosage alteration was similar in normozoospermic and non-normozoospermic TGCT patients $(10.4 \%$ vs. $10.0 \%)$ and in seminoma $(10.8 \%)$ vs. non-seminoma patients $(9.0 \%)$. The highest frequency was observed in normozoospermic seminoma patients (11.7\%) (Table 1).

\section{Comparison of the phenotypic characteristics between TGCT patients with and without gr/gr deletion, partial AZFC duplications, and DAZ dosage alteration}

Genotype/phenotype correlation is reported in Table 3. For all AZFc rearrangements the most frequent histology was seminoma both in carriers and non-carriers ranging between 70 and $78 \%$. The normozoospermic phenotype was more frequently encountered in $g r / g r$ deletion carriers $(64.3 \%)$ than in non-carriers (53.6\%). On the contrary, partial AZFc duplication carriers were more frequently nonnormozoospermic than non-carriers $(59.1 \%$ vs. $45.8 \%$, respectively). No statistical differences were found between carriers and non-carriers of the distinct rearrangements as far as testis volume, hormonal dosage values and routine sperm parameters are concerned (Table 3 and Supplementary Table 1). History of cryptorchidism was more frequent in TGCT patients with $g r / g r$ deletion than in those without deletion $(27.3 \%$ vs. $15 \%$; $p=0.27)$. History of cryptorchidism was absent in partial AZFc duplications. No familial cases of TGCT and history of hypospadias were reported in carriers of the distinct rearrangements.

\section{Comparison of the frequency of AZFc rearrangements between TGCT patients and controls}

\section{Partial AZFc deletions}

The frequency of all partial AZFc deletions carriers was higher in our TGCT cohort than in the control group (3.8\% vs. $2.5 \%)$, without reaching statistical significance
(Table 4). Partial AZFc deletions confer significant TGCT risk in the following comparisons performed against the normozoospermic controls: i) the entire TGCT group $(\mathrm{OR}=3.5,95 \% \mathrm{CI}=1.7-7.3, p=0.001)$; ii) normozoospermic TGCT $(\mathrm{OR}=4.1,95 \% \mathrm{CI}=1.8-9.3, p=0.001)$. In addition, partial $\mathrm{AZFc}$ deletions were observed more frequently in non-normozoospermic TGCT compared to normozoospermic controls ( $3.1 \%$ vs. $1.1 \%)$, although not reaching statistical significance (Table 4).

1) gr/gr deletion: although the frequency of this deletion type was higher in TGCT patients than in the control group $(2.8 \%$ vs. $2.0 \%)$, this difference was not statistically significant (Table 4). It is worth noticing that, in the control cohort, non-normozoospermic patients presented a significantly higher frequency (3.1\%) compared to normozoospermic subjects $(0.9 \%)(\mathrm{OR}=3.4 ; 95 \% \mathrm{CI}=1.7-7.0$; $p=0.000$ ). The comparison of the deletion frequency between the entire TGCT group (2.8\%) and the normozoospermic control group $(0.9 \%)$ shows a 3 -fold increased risk for TGCT in the deletion carriers $(\mathrm{OR}=3.1 ; 95 \% \mathrm{CI}=$ $1.4-7.0 ; p=0.006$ ) (Table 4). An even higher risk was observed when normozoospermic TGCT patients (3.4\%) were compared to normozoospermic controls $(0.9 \%)$ $(\mathrm{OR}=3.7 ; 95 \% \mathrm{CI}=1.5-9.1 ; p=0.006)$ (Table 4).

2) $b 2 / b 3, b 1 / b 3$, and atypical deletions: the frequency of these deletions was higher in the TGCT group (1\%) in respect to the controls $(0.5 \%)$ but without reaching statistical significance, $p=0.191$.

\section{Partial AZFc duplications}

These rearrangements were observed in 22 TGCT patients $(4.6 \%)$, while they were detected in 51/1373 controls (3.7\%). We did not found significant differences between the TGCT group and the control group, even when the comparison was done in subgroups based on seminal parameters (Table 4).

\section{DAZ dosage alteration}

The frequency was higher in TGCT patients than in the control group $(10.3 \%$ vs. $7.8 \% p=0.056)$. It is worth noticing that in the control cohort, non-normozoospermic patients presented a significantly higher frequency $(9.9 \%)$ compared to normozoospermic subjects $(6.0 \%)(\mathrm{OR}=1.7$; $95 \% \mathrm{CI}=1.2-2.6 ; \quad p=0.004)$. The dosage alteration frequency between the entire TGCT group $(10.3 \%)$ and the normozoospermic control group was statistically significantly higher $(\mathrm{OR}=1.8 ; 95 \% \mathrm{CI}=1.2-2.7 ; p=0.004)$ (Table 4). The comparison of normozoospermic controls vs. normozoospermic TGCT patients showed that $D A Z$ dosage alteration confers a significant TGCT risk $(\mathrm{OR}=1.8$; $95 \% \mathrm{CI}=1.1-3.0 ; p=0.013$ ) (Table 4). Concerning the 
Table 3 Comparison of the phenotypic characteristics between TGCT patients with and without $g r / g r$ deletion, partial AZFc duplications and DAZ dosage alteration. a) Histology and semen phenotype; b) testis volume, hormonal dosage and clinical risk factors. The frequency between subgroups did not result significantly different for any of the analyzed parameters

\begin{tabular}{|c|c|c|c|c|c|c|c|c|}
\hline \multirow[t]{2}{*}{ (a) } & & \multicolumn{3}{|c|}{$g r / g r$ deletion } & \multicolumn{2}{|c|}{ Partial AZFc duplications } & \multicolumn{2}{|c|}{$D A Z$ dosage alteration } \\
\hline & & \multicolumn{2}{|c|}{ No $(n=483)$} & Yes $(n=14)$ & No $(n=456)$ & Yes $(n=22)$ & No $(n=446)$ & Yes $(n=51)$ \\
\hline \multicolumn{9}{|l|}{ Histology } \\
\hline Seminoma & \multicolumn{3}{|c|}{$342(70.8 \%)$} & $11(78.6 \%)$ & $322(70.6 \%)$ & $16(72.7 \%)$ & $315(70.6 \%)$ & $38(74.5 \%)$ \\
\hline Non-seminoma & \multicolumn{3}{|c|}{$141(29.2 \%)$} & $3(21.4 \%)$ & $134(29.4 \%)$ & $6(27.3 \%)$ & $131(29.4 \%)$ & $13(25.5 \%)$ \\
\hline \multicolumn{9}{|c|}{ Semen parameters. Median value (minimum-maximum) } \\
\hline Volume (ml) & \multicolumn{3}{|c|}{$3.2(0.3-10.5)$} & $3.6(1.8-5.9)$ & $3.2(0.3-10.5)$ & $3.0(1.0-5.9)$ & $3.2(0.3-10.5)$ & $3.2(1.0-5.9)$ \\
\hline Sperm Concentration $\left(10^{6}\right.$ & \multicolumn{3}{|c|}{$14.9(0-336.0)$} & $30.0(0-100.0)$ & $15.0(0-336.0)$ & $7.0(0-125.0)$ & $15.0(0-336.0)$ & $21.0(0-125.0)$ \\
\hline Total sperm count $\left(10^{6}\right)$ & \multicolumn{3}{|c|}{$47.3(0-915.9)$} & $94.2(0-720.0)$ & $48.3(0-915.9)$ & $30.0(0-375.0)$ & $46.5(0-915.9)$ & $63.0(0-720.0)$ \\
\hline Progressive motility (\%) & \multicolumn{3}{|c|}{$47.0(0-81.0)$} & $44.0(0-84.0)$ & $47.0(0-81.0)$ & $41.0(0-69.0)$ & $47.0(0-81.0)$ & $40.5(0-84.0)$ \\
\hline Total motile sperm count & \multicolumn{3}{|c|}{$19.6(0-650.3)$} & $41.2(0-316.8)$ & $19.6(0-650.3)$ & $10.3(0-258.8)$ & $19.4(0-650.3)$ & $37.4(0-316.8)$ \\
\hline Normal morphology (\%) & \multicolumn{3}{|c|}{$8.0(0-47.0)$} & $7.0(0-35.0)$ & $8.0(0-47.0)$ & $6.0(0-33.0)$ & $8.0(0-47.0)$ & $6.0(0-35.0)$ \\
\hline \multicolumn{9}{|l|}{ Semen phenotype } \\
\hline Normozoospermic & & $259\left(53.6^{c}\right.$ & & $9(64.3 \%)$ & $247(54.2 \%)$ & $9(40.9 \%)$ & $240(53.8 \%)$ & $28(54.9 \%)$ \\
\hline Non-normozoospermic & & $224\left(46.4^{c}\right.$ & & $5(35.7 \%)$ & $209(45.8 \%)$ & $13(59.1 \%)$ & $206(46.2 \%)$ & $23(45.1 \%)$ \\
\hline (b) & $g r / g$ & deletion & & & Partial AZFc dupl & ications & $D A Z$ dosage alte & ation \\
\hline & No & $n=361)$ & Yes & $(n=11)$ & No $(n=348)$ & Yes $(n=11)$ & No $(n=338)$ & Yes $(n=34)$ \\
\hline Testis volume (ml), Median & lue ( & minimum-me & ximu & & & & & \\
\hline Right testis (ml) & 20.0 & $(8.0-30.0)$ & $22 .($ & $(18.0-30.0)$ & $20.0(8.0-30.0)$ & $17.5(8.0-25.0)$ & $20.0(8.0-30.0)$ & $18.0(8.0-30.0)$ \\
\hline Left testis (ml) & 20.0 & $(7.0-30.0)$ & $19 .($ & $(8.0-25.0)$ & $19.0(7.0-30.0)$ & $20.0(12.0-25.0)$ & $20.0(7.0-30.0)$ & $19.5(8.0-25.0)$ \\
\hline Hormonal analysis. Median & lue ( & minimum-ma & ximu & & & & & \\
\hline LH (mU/ml) & 5.9 & $(1.9-25.5)$ & & $(1.5-11.2)$ & $6.1(1.9-25.5)$ & $5.0(2.4-8.0)$ & $5.9(1.9-22.5)$ & $5.5(1.5-25.5)$ \\
\hline FSH (mU/ml) & 9.7 & $(1.4-45.7)$ & & (3.7-28.6) & $9.9(1.4-45.7)$ & $7.2(2.3-12.9)$ & $9.9(1.4-45.7)$ & $7.2(1.5-42.2)$ \\
\hline Testosterone $(\mathrm{nmol} / \mathrm{l})$ & 14.1 & $(4.4-33.6)$ & 18.8 & $(15.8-24.8)$ & $14.1(4.4-33.6)$ & $13.1(5.9-23.1)$ & $14.3(4.4-33.6)$ & $14.8(5.9-24.8)$ \\
\hline Family history of TGCT & & $(1.1 \%)$ & & $(0.0 \%)$ & $4(1.2 \%)$ & $0(0 \%)$ & $4(1.2 \%)$ & $0(0 \%)$ \\
\hline Cryptorchidism & & $(15.0 \%)$ & & $(27.3 \%)$ & $53(15.2 \%)$ & $0(0 \%)$ & $53(15.7 \%)$ & $4(11.8 \%)$ \\
\hline Hypospadias & & $(0.8 \%)$ & & $(0.0 \%)$ & $3(0.9 \%)$ & $0(0 \%)$ & $3(0.9 \%)$ & $0(0 \%)$ \\
\hline
\end{tabular}

${ }^{a}$ Patients with a choriocarcinoma component in their testis histology were excluded $(n=29)$

non-normozoospermic TGCT patients, a higher frequency of the $D A Z$ dosage alteration in this subgroup of patient vs. normozoospermic control group was found, with a $p$-value close to significance $(p=0.027)$ (Table 4$)$.

\section{Discussion}

Testicular germ cell tumors (TGCTs) derive from a preinvasive precursor lesion, called germ cell neoplasia in situ (GCNIS) and show an incidence that peaks in young adulthood [1, 2]. Basic research studies and epidemiological trends support the hypothesis that TGCT is of fetal origin and it is due to failure of normal fetal programming of the differentiation of primordial germ cells through a gonocyte stage into spermatogonia [2]. The differentiation process of primordial germ cells/gonocytes is regulated through several germ cell-specific genes including $\mathrm{Y}$ chromosomelinked ones. The alteration of germ cell differentiation might set the basis both for the development of testicular tumour and for impaired spermatogenesis.

The AZF genes on the long arm of the $\mathrm{Y}$ chromosome are important regulators of spermatogenesis and genes belonging to the AZFc region are specifically expressed in male germ cells. The most frequent, known molecular genetic cause of oligo/azoospermia are complete AZF deletions [14] and their role in TGCT has been ruled out by previous studies [16-18, 21]. On the contrary, the role of $g r /$ $g r$ deletion (removing half of the AZFc gene content) is still debated due to contrasting results in the literature [21-23]. The largest multiethnic study by Nathanson et al. [22] tested for the $g r / g r$ deletion in 1842 TGCT cases and 2599 
unaffected men. TGCT cases were significantly more likely to carry the $g r / g r$ deletion compared to unaffected males $(\mathrm{OR}=2.1 ; 95 \% \mathrm{CI}=1.3-3.6 ; p=0.005)$ and this association was stronger in men with seminoma than for those with non-seminoma. In a subsequent study [23], which evaluated 263 TGCT cases (167 with family history and 96 sporadic cases), the authors were not able to demonstrate a significant association neither for $g r / g r$ deletion nor for other rare partial AZFc deletions. In these two studies the fertility status and/or sperm parameter of the patients were not available. The only study in which semen parameters were reported is based on a small Italian cohort $(n=118)$ in which no $g r / g r$ deletion carriers were found among TGCT patients [21]. Hence, it remains to be established whether the association between $g r / g r$ deletion and TGCT is restricted to a specific seminal subgroup of patients (subjects with reduced sperm count).

Our study has been designed to evaluate the role of partial AZFc deletions/duplications and specifically the $D A Z$ gene dosage variation in TGCT in the largest European study population available to date.

Our first objective was to elucidate the role of $g r / g r$ deletion as genetic risk factor for TGCT in two Mediterranean populations with full semen/andrological characterization. When dealing with Y chromosome-related risk factors, matching for ethnicity and geographic origin is fundamental in order to avoid population stratification bias [29]. Consequently, much care was taken for geographic and ethnic matching in our study. Semen parameters were known both for cases and controls, allowing us to evaluate the role of $g r / g r$ deletion as TGCT genetic risk factor in combination with spermatogenic efficiency. The similar frequency of $g r / g r$ deletion in the TGCT group (2.8\%) and the entire control group (2.0\%) is due to the relatively high frequency of $g r / g r$ deletion in patients with idiopathic oligo/ azoospermia (3.1\%). In fact, in our previous studies we demonstrated that $g r / g r$ deletion is significantly associated with oligozoospermia in the Spanish and Italian populations $[13,27,30]$. Surprisingly enough, our most important finding is related to the normozoospermic group: while only $0.9 \%$ of controls were carrier of $g r / g r$ deletion, this percentage was higher in normozoospermic TGCT patients (3.4\%) and even higher in normozoospermic seminoma patients (4.1\%). $g r / g r$ deletion in normozoospermic subjects confers almost 4-fold increased risk for developing testis tumor.

The specific genes removed can vary between $g r / g r$ deletions, and this has been suggested as the most direct modulating factor for the associated semen phenotype [13]. In this regard, mainly the $D A Z$ and $C D Y 1$ copies, which lie within the deleted AZFc region, have been examined as possible predictors for pathogenicity. The loss of DAZ1/ $D A Z 2$ and $C D Y 1 \mathrm{~A}$ has been proposed as more deleterious 
than the removal of $D A Z 3 / D A Z 4$ and $C D Y 1 B$ [26, 31, 32], however this was not fully proved by a large multicenter study [33]. Concerning $D A Z$ gene copies, different members of the $D A Z$ gene family have different number of RNA recognition motifs (RRMs) and $D A Z$ repeats, which may confer different functional activity to the four $D A Z$ copies [33]. Hence, we were interested in investigating on the deletion "haplotypes" (based on different combinations of $D A Z$ and $C D Y 1$ copies) in TGCT patients with and without altered spermatogenesis and with different tumor histology. Moreover, we questioned whether the deletion haplotypes in TGCT patients were different from that of the controls especially concerning the deleted $D A Z$ copies. 11 (78.6\%) patients presented a $C D Y 1 A$ deletion, and data break-down showed that this type of deletion was the most frequent in all semen and histology phenotypes. A similarly high frequency of $C D Y 1 A$ deletion was found in the control group (77.8\%). Concerning the subtype of $D A Z$ gene copy deletion, there was no predominant copy loss in specific sperm count-based subgroups or histology types. The same finding was observed in the control group. This data indicates that the type of deleted $C D Y 1$ or $D A Z$ copies are irrelevant to sperm output or histology type in TGCT patients carrying the $g r / g r$ deletion. Most importantly, the deletion "haplotype" was not different between TGCT cases and tumourfree controls.

Beside $g r / g r$ deletions, three other types of deletions were identified in our cohorts $(b 2 / b 3, b 1 / b 3$ and atypical deletions). The frequency of these deletions was extremely rare both in patients and controls $(0.6 \%$ vs. $0.4 \%, 0.2 \%$ vs. $0.0 \%, 0.2 \%$ vs. $0.2 \%$, respectively), hence their role, if any, in TGCT remains to be established.

Given that partial AZFc deletions perturb AZFc gene dosage, we were interested in analyzing the role of an increased gene dosage ( $>4$ for $D A Z$ and $>2$ for $C D Y 1$, respectively) due to partial duplications. Although case/ control association studies failed to reach to a univocal conclusion in respect to the hypothetical "optimal" AZFc gene dosage needed for normal spermatogenesis $[27,30,34,35]$, it seems that a positive selection for an "optimal" gene dosage does exist (four $D A Z$ and two $C D Y 1$ copies) during evolution [36]. Hence, our working hypothesis was a possible predisposing effect of AZFc dosage variation (not only deficit but also an excess) to TGCT development. Partial AZFc duplications frequency was $4.6 \%$ in TGCT patients while it was $3.7 \%$ in controls. Therefore, on the contrary of $g r / g r$ deletion, we were unable to detect a significant effect of partial AZFc duplications on TGCT development.

We have separately evaluated the $D A Z$ gene dosage variation (as a consequence of partial AZFc deletions, duplications or isolated $D A Z$ duplication event). We found that patients with TGCT have a higher frequency of alterations of the $D A Z$ dosage with respect to the entire control group (10.3\% vs. $7.8 \%$ ) (Table 4). The comparison of the entire TGCT group vs. normozoospermic controls showed a significant difference $(10.3 \%$ vs. $6.0 \%, p=0.004)$ indicating that $D A Z$ dosage alteration in normozoospermic subjects confers almost 2 -fold increased risk for developing TGCT $(\mathrm{OR}=1.8,95 \% \mathrm{CI}=1.2-2.7)$.

The biological link between AZFc gene dosage variation (especially its reduction due to deletions) and tumourigenesis remains elusive. One hypothesis could be that $D A Z$ gene dosage may influence the differentiation of the gonocytes. In fact, it has been demonstrated that $D A Z$ is present in both the nuclei and cytoplasm of fetal gonocytes and in spermatogonial nuclei [37]. Hence, based on its expression profile, $D A Z$ gene dosage variation may play a role in TGCT etiology. Alternatively, we can also speculate that the "fragility" expressed as deletions/duplications of the $\mathrm{Y}$ chromosome is a marker for general "genomic instability" potentially leading to tumors, including TGCT.

In conclusion, our data provide evidence that the $g r / g r$ deletion is a risk factor for TGCT also in the Italian and Spanish populations and that normozoospermic $\mathrm{gr} / \mathrm{gr}$ deletion carriers have almost 4-fold increased risk to develop TGCT. The observed significant association between $g r / g r$ deletion and normozoospermic TGCT, suggests that this deletion is an independent risk factor from impaired spermatogenesis. In line with this finding, a recent epidemiological study reported an increased risk for TGCT in first-degree relatives of subfertile normozoospermic men [38]. Diagnosing $g r / g r$ deletion in a TGCT patient has a potential clinical relevance to his male relatives, especially to the brothers in whom preventive measures such as regular autopalpation or ultrasound scan of the testis can be proposed. In some countries, $g r / g r$ deletion screening is already part of the routine genetic diagnosis of oligozoospermic men [14]. This implies that similarly to brothers of $g r / g r$ deletion carriers, also infertile patients diagnosed with this type of deletion should receive careful long-term andrological follow-up.

Acknowledgements This paper is in remembrance of Prof. Loredana Gandini, who actively participated in conducting the study but passed away before its completion. The work was supported by the following funding agencies: the Spanish Ministry of Health, Instituto Carlos III-FIS (FIS/FEDER-PI14/01250 and PI17/01822), Fundación Merck Salud (Ayudas Merck de Investigación 2017 en el área de Investigación Clínica en Fertilidad) awarded to C.K. and the Istituto Toscano Tumori grant awarded to C.K.; the European Commission, Reproductive Biology Early Research Training (REPROTRAIN, Project Number: 289880) awarded to C.K. and A.R.E. The authors wish to thank the patients participating in the study for their important collaboration. We also thank the clinicians from the Andrology Unit of the Fundació Puigvert, J. Sarquella, A. Vives and Ll. Bassas. We acknowledge Esperança Martí, President of the Fundació Puigvert. 
Author contributions Conceived and designed the experiments: C.K. Patient recruitment and their clinical characterization: D.M.M., D.P., A.M., J.S.C., F.L., A.L. Performed the experiments D.M.M., E.C., C.C., A.R.E., C.G., M.G.F., S.V., F.C. Analyzed the data: D.M.M., E.C., C.K. Contributed reagents/materials/analysis tools: C.K., D.P., F.L., E.R.C., E.A. Wrote the paper: C.K., D.M.M., E.C.

\section{Compliance with ethical standards}

Conflict of interest The authors declare that they have no conflict of interest.

Ethical approval All patients were consented for collection of data. The local ethical committees of the University Hospital Careggi, Fundació Puigvert and "Sapienza" University of Rome approved the study. The study was performed in accordance with the Declaration of Helsinki.

Publisher's note: Springer Nature remains neutral with regard to jurisdictional claims in published maps and institutional affiliations.

\section{References}

1. Znaor A, Lortet-Tieulent J, Jemal A, Bray F. International variations and trends in testicular cancer incidence and mortality. Eur Urol. 2014;65:1095-106.

2. Rajpert-De Meyts E, Skakkebaek NE, Toppari J. Testicular cancer pathogenesis, diagnosis and endocrine aspects. South Dartmouth, MA: MDText.com, Inc.; 2000-2018.

3. Looijenga LH. Human testicular (non)seminomatous germ cell tumours: the clinical implications of recent pathobiological insights. J Pathol. 2009;218:146-62.

4. Krausz C, Looijenga LH. Genetic aspects of testicular germ cell tumours. Cell Cycle. 2008;7:3519-24.

5. Litchfield K, Levy M, Huddart RA, Shipley J, Turnbull C. The genomic landscape of testicular germ cell tumours: from susceptibility to treatment. Nat Rev Urol. 2016;13:409-19.

6. Hemminki K, Li X. Familial risk in testicular cancer as a clue to a heritable and environmental aetiology. Br J Cancer. 2004;90: 1765-70.

7. Kharazmi E, Hemminki K, Pukkala E, Sundquist K, Tryggvadottir $\mathrm{L}$, Tretli $\mathrm{S}$, et al. Cancer risk in relatives of testicular cancer patients by histology type and age at diagnosis: A Joint Study from Five Nordic Countries. Eur Urol. 2015;68:283-9.

8. Gajendran VK, Nguyen M, Ellison LM. Testicular cancer patterns in African-American men. Urology . 2005;66:602-5.

9. Litchfield K, Thomsen H, Mitchell JS, Sundquist J, Houlston RS, Hemminki K, et al. Quantifying the heritability of testicular germ cell tumour using both population-based and genomic approaches. Sci Rep. 2015;5:13889.

10. Skakkebaek NE, Rajpert-De Meyts E, Main KM. Testicular dysgenesis syndrome: an increasingly common developmental disorder with environmental aspects. Hum Reprod. 2001;16:972-8.

11. Hotaling JM, Walsh TJ. Male infertility: a risk factor for testicular cancer. Nat Rev Urol. 2009;6:550-6.

12. Elzinga-Tinke JE, Sirre ME, Looijenga LH, van Casteren N, Wildhagen MF, Dohle GR. The predictive value of testicular ultrasound abnormalities for carcinoma in situ of the testis in men at risk for testicular cancer. Int J Androl. 2010;33:597-603.

13. Krausz C, Casamonti E. Spermatogenic failure and the Y chromosome. Hum Genet. 2017;136:637-55.

14. Krausz C, Hoefsloot L, Simoni M, Tüttelmann F. European Academy of Andrology; European Molecular Genetics Quality Network. EAA/EMQN best practice guidelines for molecular diagnosis of Y-chromosomal microdeletions: state-of-the-art 2013. Andrology . 2014;2:5-19.

15. Repping S, Skaletsky H, Brown L, van Daalen SK, Korver CM, Pyntikova T, et al. Polymorphism for a 1.6-Mb deletion of the human Y chromosome persists through balance between recurrent mutation and haploid selection. Nat Genet. 2003;35:247-51.

16. Bor $\mathrm{P}$, Hindkjaer J, Kølvraa S, Rossen $\mathrm{P}$, von der Maase $\mathrm{H}$, Jørgensen TM, et al. Screening for Y microdeletions in men with testicular cancer and undescended testis. J Assist Reprod Genet. 2006;23:41-5.

17. Lutke Holzik MF, Storm K, Sijmons RH, D'hollander M, Arts EG, Verstraaten ML, et al. Absence of constitutional Y chromosome AZF deletions in patients with testicular germ cell tumours. Urology. 2005;65:196-201.

18. Frydelund-Larsen L, Vogt PH, Leffers H, Schadwinkel A, Daugaard G, Skakkebaek NE, et al. No AZF deletion in 160 patients with testicular germ cell neoplasia. Mol Hum Reprod. 2003;9:517-21.

19. Quintana-Murci L, Weale ME, Thomas MG, Erdei E, Bradman N, Shanks JH, et al. Y chromosome haplotypes and testicular cancer in the English population. J Med Genet. 2003;40:e20.

20. Ewis AA, Lee J, Naroda T, Kagawa S, Baba Y, Nakahori Y. Lack of association between the incidence of testicular germ cell tumours and Y-chromosome haplogroups in the Japanese population. Int J Urol. 2006;13:1212-7.

21. Ferlin A, Speltra E, Garolla A, Selice R, Zuccarello D, Foresta C. Y chromosome haplogroups and susceptibility to testicular cancer. Mol Hum Reprod. 2007;13:615-9.

22. Nathanson KL, Kanetsky PA, Hawes R, Vaughn DJ, Letrero R, Tucker K, et al. The Y deletion gr/gr and susceptibility to testicular germ cell tumour. Am J Hum Genet. 2005;77:1034-43.

23. Linger R, Dudakia D, Huddart R, Easton D, Bishop DT, Stratton $\mathrm{MR}$, et al. A physical analysis of the $\mathrm{Y}$ chromosome shows no additional deletions, other than $\mathrm{Gr} / \mathrm{Gr}$, associated with testicular germ cell tumour. Br J Cancer. 2007;96:357-61.

24. World Health Organization. WHO laboratory manual for the Examination and processing of human semen. Geneva: WHO Press; 2010.

25. Miller SA, Dykes DD, Polesky HF. A simple salting out procedure for extracting DNA from human nucleated cells. Nucleic Acids Res. 1988;16:1215.

26. Giachini C, Guarducci E, Longepied G, Degl'Innocenti S, Becherini L, Forti G, et al. The gr/gr deletion(s): a new genetic test in male infertility? J Med Genet. 2005;42:497-502.

27. Giachini C, Laface I, Guarducci E, Balercia G, Forti G, Krausz C. Partial AZFc deletions and duplications: clinical correlates in the Italian population. Hum Genet. 2008;124:399-410.

28. Karafet TM, Mendez FL, Meilerman MB, Underhill PA, Zegura SL, Hammer MF. New binary polymorphisms reshape and increase resolution of the human $\mathrm{Y}$ chromosomal haplogroup tree. Genome Res. 2008;18:830-8.

29. Tyler-Smith C. An evolutionary perspective on Y-chromosomal variation and male infertility. Int J Androl. 2008;31:376-82.

30. Lo Giacco D, Chianese C, Sánchez-Curbelo J, Bassas L, Ruiz P, Rajmil O, et al. Clinical relevance of Y-linked CNV screening in male infertility: new insights based on the 8-year experience of a diagnostic genetic laboratory. Eur J Hum Genet. 2014;22:754-61.

31. Fernandes S, Huellen K, Goncalves J, Dukal H, Zeisler J, Rajpert De Meyts E, et al. High frequency of DAZ1/DAZ2 gene deletions in patients with severe oligozoospermia. Mol Hum Reprod. 2002;8:286-98.

32. Ferlin A, Tessari A, Ganz F, Marchina E, Barlati S, Garolla A, et al. Association of partial AZFc region deletions with spermatogenic impairment and male infertility. J Med Genet. 2005;42: 209-13. 
33. Krausz C, Giachini C, Xue Y, O’Bryan MK, Gromoll J, Rajpertde Meyts E, et al. Phenotypic variation within European carriers of the Y-chromosomal gr/gr deletion is independent of Ychromosomal background. J Med Genet. 2009;46:21-31.

34. Lin YW, Hsu LC, Kuo PL, Huang WJ, Chiang HS, Yeh SD, et al. Partial duplication at $\mathrm{AZFc}$ on the $\mathrm{Y}$ chromosome is a risk factor for impaired spermatogenesis in Han Chinese in Taiwan. Hum Mutat. 2007;28:486-94.

35. Lu C, Jiang J, Zhang R, Wang Y, Xu M, Qin Y, et al. Gene copy number alterations in the azoospermia-associated $\mathrm{AZFc}$ region and their effect on spermatogenic impairment. Mol Hum Reprod. 2014;20:836-43.
36. Teitz LS, Pyntikova T, Skaletsky H, Page DC. Selection has countered high mutability to preserve the ancestral copy number of $\mathrm{Y}$ chromosome amplicons in diverse human lineages. Am $\mathbf{J}$ Hum Genet. 2018;103:261-75.

37. Reijo RA, Dorfman DM, Slee R, Renshaw AA, Loughlin KR, Cooke $\mathrm{H}$, et al. DAZ family proteins exist throughout male germ cell development and transit from nucleus to cytoplasm at meiosis in humans and mice. Biol Reprod. 2000;63:1490-6.

38. Anderson RE, Hanson HA, Patel DP, Johnstone E, Aston KI, Carrell DT, et al. Cancer risk in first- and seconddegree relatives of men with poor semen quality. Fertil Steril. 2016;106:731-8. 